\title{
The Magnetic Control Adjustable Characteristics of Transmission Spectrum of One-dimensional Thue-Morse Quasiperiodic Structure with Nanoparticle Magnetic Fluid
}

\author{
LI Jiaoping ${ }^{1, a}$, XIE Yingmao ${ }^{2, b^{*}}$, HAO Lili ${ }^{1, c}$, LIAO Kun ${ }^{1, d}$
}

\author{
${ }^{1}$ School of Physics and Electronic Information, Gannan Normal University, Ganzhou 341000, China \\ ${ }^{2}$ Institute of Optoelectronic Materials and Technology, Gannan Normal University, Ganzhou 341000, \\ China
}

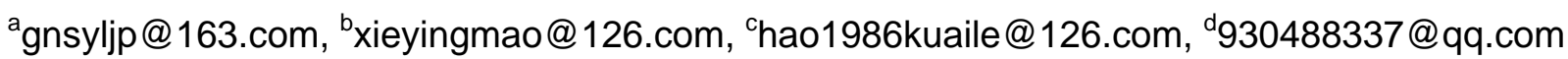

Keywords: Quasiperiodic Structure; Magnetic Fluid; Photonic Crystals; Transmission Spectrum; Transfer Matrix Method

\begin{abstract}
The magnetic control adjustable characteristics of one-dimensional Thue-Morse quasiperiodic structure with nanoparticle magnetic fluid defect local modes are studied by using the transfer matrix method. First, the physical model of one-dimensional Thue-Morse quasiperiodic structure with nanoparticle magnetic fluid photonic crystal is built. Then, the transmission spectrum of one-dimensional Thue-Morse quasiperiodic structure with nanoparticle magnetic fluid is calculated by transfer matrix method. Finally, the transmission spectrum of magnetic control adjustable characteristics is discussed. The results show that the wavelength of the defect local modes shift to the long-wave direction with the refractive index of magnetic fluid increasing; The wavelength drift variation increases as the level of the defect local modes at the different level increase; The quality factor decreases with the increase of the refractive index for the defect local modes at the same level.
\end{abstract}

\section{Introduction}

In 1984, Shechtman and others published the report about study of the quasicrystal for the first time [1]. It has attracted widespread attention and becomes the research hotspot of materials science [2]. Quasicrystal structure is aperiodic structure, which is the structure between periodic structure and disordered structure at the structural features. Compared to periodic structure, aperiodic structure has no translation symmetry; Compared to disordered structure, aperiodic structure is the system of ordering and certainty. Quasiperiodic structure is a kind of the particular aperiodic structure. The typical quasiperiodic structures are Fibonacci, Cantor and Thue-Morse [2], etc.

Thue-Morse quasiperiodic structure has attracted scientists to study in detail, because it is a kind of typical quasiperiodic structure. The Thue-Morse sequence can be defined by the recursive relations: $S_{n}=S_{n-1} S^{+}{ }_{n-1}$ and $S_{n}^{+}=S_{n-1}^{+} S_{n-1}(n \geq 1)$. If you start from $A$, the former five dynasties of Thue-Morse generations can be written as [2]

$$
S_{0}=\{A\}, S_{1}=\{A B\}, S_{2}=\{A B B A\}, S_{3}=\{A B B A B A A B\}, S_{4}=\{A B B A B A A B B A A B A B B A\} .
$$

Photonic crystal with nanoparticle magnetic fluid is a kind of tunable photonic crystal [3] [4]. Because the photonic crystal with nanoparticle magnetic fluid has non-contact magnetic control adjustable features, which has attracted application prospect on the new magnetron tunable photonic devices, causing the attention of the relevant researchers both at home and abroad, and it has became a hot spot in the field of a new tunable photonic crystal. There are scholars who study the magnetic field control characteristics of photonic crystal fiber. The magnetic field sensor is made. For example, the research team of Nanyang technological university in Singapore fills the nanoparticle magnetic fluid of higher refractive index $(>1.45)$ in the hollow of magnetic photonic crystal fiber, based on the band gap effect of the photonic crystal fiber with magnetic fluid which studies the magnetic fiber optic sensor [5]. The colloid ferrofluid as primitive materials of one-dimensional photonic crystals by the team of Ji-ping Huang at Fudan University, which studies 
the magnetic control of one-dimensional photonic crystals based on colloid ferrofluid [6] [7]. In 2013, the bandgap management in Two-dimensional photonic crystal Thue-Morse structures are studied by Mehdizadeh and others [8]. In 2014, the transmission spectrum of electrons through a Thue-Morse graphene superlattice is studied by Korol [9].

However, there is rarely report for the study of one-dimensional Thue-Morse quasiperiodic structures with nanoparticle magnetic fluid. This paper studies the magnetic control adjustable characteristics of one-dimensional Thue-Morse quasiperiodic structure defect local modes with nanoparticle magnetic fluid. Firstly, the physical model of one-dimensional Thue-Morse quasiperiodic structure with nanoparticle magnetic fluid photonic crystal is built. Then, the transmission spectrum of one-dimensional Thue-Morse quasiperiodic structure with nanoparticle magnetic fluid is calculated by transfer matrix method. Finally, the transmission spectrum of magnetic control adjustable features is discussed.

\section{Physical Model}

The physical model of one-dimensional Thue-Morse quasiperiodic structure with nanoparticle magnetic fluid photonic crystal is as shown in Figure 1. It shows that the fourth generation structure, $S_{4}=\{A B B A B A A B B A A B A B B A\}$. A is Si dielectric layer that refractive index is $n_{a}=3.42$, and B is dielectric layer of magnetic fluid.

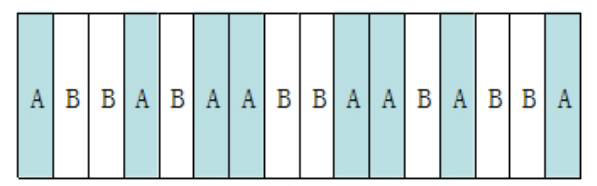

Fig.1. The physical model of one-dimensional Thue-Morse quasiperiodic structure with nanoparticle magnetic fluid photonic crystal

\section{The Results of Numerical Calculation and Discussion}

The Primitive materials of one-dimensional Thue-Morse quasiperiodic structure are $\mathrm{Si}$ and water-based $\mathrm{Fe}_{3} \mathrm{O}_{4}$ magnetic fluids, the optical thickness is equal, $n_{a} * d_{a}=\overline{n_{b}} * d_{b}=\lambda_{0} / 4, \lambda_{0}$ is center wavelength. The refractive index $n_{b}$ of magnetic liquid is closely related to the magnetic liquid distribution state of the magnetic particles. The relative influence factors of distribution of the magnetic particles in magnetic fluids are: the magnetic dipole interaction between particles, the Brownian motion of particles which is closely related to the temperature, extra magnetic field, capture light, etc. When the temperature of room is $20{ }^{\circ} \mathrm{C}$, wavelength of light source is $1550 \mathrm{~nm}$, density of water-based of $\mathrm{Fe}_{3} \mathrm{O}_{4}$ magnetic field is $1.2 \mathrm{~g} / \mathrm{mL}$, the range of the extra magnetic field intensity variation from 0 0e to $16610 \mathrm{e}$, the refractive index $n_{b}$ of magnetic liquid decreases from $n_{b h}=1.447$ to $n_{b l}=1.425$ with the increase of magnetic field intensity. Here, water-based $\mathrm{Fe}_{3} \mathrm{O}_{4}$ magnetic fluid as the primitive materials of photonic crystals is selected.

The refractive index of dielectric layers $\mathrm{A}$ and $\mathrm{B}$ are ${ }^{n_{a}},{ }^{n_{b}}$ respectively, and its thickness are $d_{a}, d_{b}$ respectively. The light wave incidents to the surface of one-dimensional Thue-Morse quasiperiodic structure with nanoparticle magnetic fluid, which is shown in figure 1 . The formulae which the light reflection and transmission coefficient is calculated by the transfer matrix method are [10]

$$
r=\frac{n_{0}\left(m_{11}+n_{N+1} m_{12}\right)-\left(m_{21}+n_{N+1} m_{22}\right)}{n_{0}\left(m_{11}+n_{N+1} m_{12}\right)+\left(m_{21}+n_{N+1} m_{22}\right)}
$$




$$
t=\frac{2 n_{0}}{n_{0}\left(m_{11}+n_{N+1} m_{12}\right)+\left(m_{21}+n_{N+1} m_{22}\right)}
$$

where $m_{i j}$ is the matrix elements of $\mathrm{M}$

$$
M_{b}=\left(\begin{array}{cc}
\cos \delta_{b} & -\frac{i}{\eta_{b}} \sin \delta_{b} \\
-i \eta_{b} \sin \delta_{b} & \cos \delta_{b}
\end{array}\right)
$$

The reflectance $\mathrm{R}$ and the transmittance $\mathrm{T}$ respectively are

$$
\begin{aligned}
& R=|r|^{2} \\
& T=|t|^{2}
\end{aligned}
$$

Taking the parameters $n_{a}=3.42, n_{b l}=1.425, n_{b h}=1.447, \overline{\mathrm{n}}_{b}=1.4385, n_{a} * d_{a}=\bar{n}_{b} * d_{b}=\lambda_{0} / 4$, the $\lambda_{0}=1550 \mathrm{~nm}$ is center wavelength. The fifth generation transmission spectrum of one-dimensional Thue-Morse quasiperiodic structure with nanoparticle magnetic fluid is calculated by transfer matrix method when the refractive index of magnetic liquid changes with the intensity of magnetic field, the results are shown in figure 2. The results show that: the wavelength of the defect local modes at the same level shift to the long-wave direction with the refractive index of magnetic fluid increases. Because the light can transmit stably in the crystal of quasiperiodic structure that must match the standing-wave conditions $n d=k \lambda, n$ is the refraction index of dielectric layer and $d$ is the thickness. So the wavelength $\lambda$ of defect local modes increases with the refractive index $n$ of defect layer increasing for the same level of defect local modes ( $k$ is certain) when the thickness $d$ is certain.

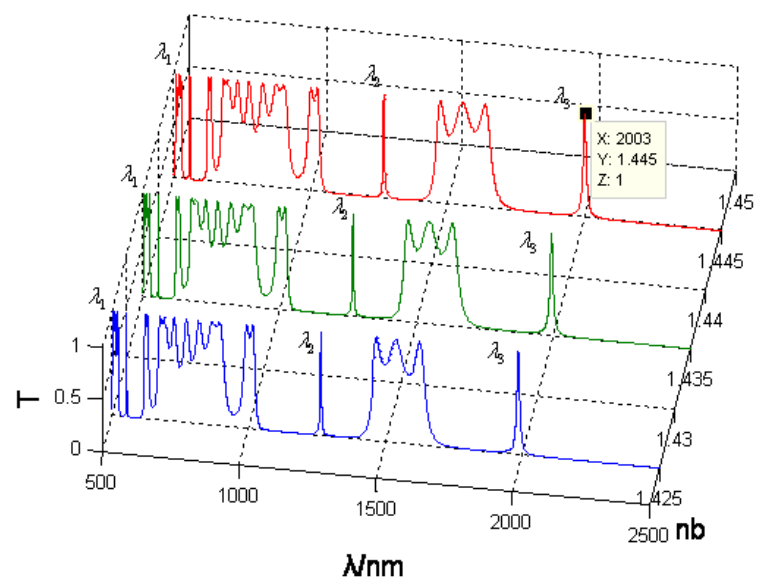

Fig.2. The transmission spectrum of one-dimensional Thue-Morse quasiperiodic structure with nanoparticle magnetic fluid

When the refractive index $n_{b}$ of water-based $\mathrm{Fe}_{3} \mathrm{O}_{4}$ magnetic liquid is different, the changing relation of defect local modes of the photonic crystal with different level is shown in table 1 . The results show that: the drift variation $\Delta \lambda$ of defect local modes $\lambda_{1}, \lambda_{2}$ and $\lambda_{3}$ are $4 \mathrm{~nm}, 8 \mathrm{~nm}, 16$ $\mathrm{nm}$ respectively when the refractive index $n_{b}$ of magnetic fluid increases from 1.425 to 1.445. Accordingly, the wavelength drift variation $\Delta \lambda$ increases with the increasing of the level for the defect local modes at the different level (the level of $\lambda_{1}<$ the level of $\lambda_{2}<$ the level of $\lambda_{3}$ ). 
Table 1 The changing relation of defect local modes of the photonic crystal with different level when the refractive index $n_{b}$ of water-based $\mathrm{Fe}_{3} \mathrm{O}_{4}$ magnetic liquid is different

\begin{tabular}{|c|c|c|c|}
\hline$n_{b}$ & $\lambda_{1} / \mathrm{nm}$ & $\lambda_{2} / \mathrm{nm}$ & $\lambda_{3} / \mathrm{nm}$ \\
\hline 1.425 & 556 & 1263 & 1987 \\
\hline 1.435 & 558 & 1267 & 1995 \\
\hline 1.445 & 560 & 1271 & 2003 \\
\hline
\end{tabular}

The quality factor of defect local modes is defined as $Q=\lambda_{0} / \Delta \lambda$, where $\lambda_{0}$ is the center wavelength, $\Delta \lambda$ is the bandwidth of half the peak. The quality factor of the defect localized modes $\lambda_{3}$ decreases from 165.58 to 154.08 , which is calculated by the figure 2 when the refractive index of magnetic fluid increases from 1.425 to 1.445. That is, the higher the refractive index of magnetic fluid is, the lower the quality factor of the defect localized mode at the same level is.

\section{Conclusion}

The transmission spectrum of one-dimensional Thue-Morse quasiperiodic structure with nanoparticle magnetic fluid is calculated by transfer matrix method. The relation on the drift variation of localized modes and the quality factor of the defect local mode at the same level with the change of the refractive index are discussed. The results show that the wavelength of the local mode shifts to the long-wave direction with increasing of the refractive index for magnetic fluid; The wavelength drift variation increases with the increasing of the level for the defect local mode at the different level; The quality factor decreases with the increasing of the refractive index for the defect local modes at the same level. That has certain guiding significance for the photonic devices such as optical switch, filter in theory.

\section{Acknowledgement}

This research was supported by the Science \& Technology Foundation for the department of education of Jiangxi Province under Grant No. GJJ13660 and the Innovation Fund Designated for Graduate Students of Jiangxi Province under Grant No.YC2014-S414.

\section{References}

[1] D. Shechtman, I. Blech, D. Gratias, et al. Metallic phase with long-range orientational order and no translational symmetry[J]. Phys. Rev. Lett., 1984, 53(20): 1951-1954.

[2] E. L. Albuquerque, M. G. Cottam. Theory of elementary excitations in quasiperiodic structures[J]. Physics Reports, 2003, 376(4): 225-337.

[3] C. Fan, G. Wang, J. Huang. Magnetocontrollable photonic crystals based on colloidal ferrofluids[J]. Journal of Applied Physics, 2008, 103(9): 094107-1-094107-3.

[4] G. Yu, S. Pu, X. Wang, H. Ji. Tunable one-dimensional photonic crystals based on magnetic fluids[J]. Optik, 2012, 124(17): 2713-2715.

[5] P. Zu, C. Chan, T. Gong, et al. Magneto-optical fiber sensor based on band gap effect of photonic crystal fiber infiltrated with magnetic fluid[J]. Appl. Phys. Lett., 2012, 101(24): 241118-1-241118-4.

[6] C. Fan. A study of the properties of several kinds of soft matter[D]: (The Doctoral Dissertation). Fudan University, 2009: 63-67.

[7] Y. Gao, C. Fan, J. Huang. The characteristics research of photonics with colloid ferrofluid[J]. Progress in physics, 2010, 30(4): 387-421.

[8] F. Mehdizadeh, H. Alipour-Banaei. Bandgap Management in Two-dimensional Photonic Crystal Thue-Morse Structures[J]. Journal of Optical Communications, 2013, 34(1): 61-65. 
[9] A. Korol. Transmission spectrum of electrons through a Thue-Morse graphene superlattice[J]. Low temperature physics: Simultaneous Russian - English publication, 2014, 40(3): 251-254.

[10] Y. Xie. A study on the Interrelated Questions for the physical Mechanism of Lasing in Random Media[D]: (The Doctoral Dissertation). Nan Chang: Nanchang University, 2007. 